\title{
Negative dielectric permittivity of nonmagnetic crystals in the terahertz waveband
}

\author{
S.G. Felinskyi ${ }^{1}$, P.A. Korotkov ${ }^{2}$, G.S. Felinskyi ${ }^{3}$ \\ Taras Shevchenko Kyiv National University, 4, prospect Glushkova, 03127 Kyiv, Ukraine \\ Phone: +380-44-526-0570; e-mail: stalisman@ukr.net ${ }^{1}$,pak@mail.univ.kiev.ua ${ }^{2}$,felinskyi@yahoo.com ${ }^{3}$
}

\begin{abstract}
Physical conditions for occurrence of the spectral bands with the negative dielectric permittivity in nonmagnetic crystalline media in the terahertz waveband have been studied in this work. It has been shown that damping the polar vibrations has a primary effect on formation of the negative dielectric permittivity in real crystals at resonance (terahertz) frequencies, and phonon attenuation imposes significant restrictions both on the frequency range and the minimum achievable value for all dielectric tensor components. Within frameworks of the single-oscillator model, the authors have obtained: i) the criterion for the existence of the negative dielectric permittivity, which is based on physical and spectroscopic parameters of the crystal, ii) analytical expressions for calculation of the frequency band where the dielectric permittivity takes negative values. Frequency regions and the minimum value of negative dielectric permittivity are quantitatively defined in the crystal $\mathrm{LiTaO}_{3}$. It is proved the applicability of the obtained relationships in cases of relatively complex phonon vibration spectra.
\end{abstract}

Keywords: negative dielectric permittivity, single-oscillator model, terahertz band, $\mathrm{LiTaO}_{3}$.

Manuscript received 29.12.11; revised version received 18.01.12; accepted for publication 26.01.12; published online 29.03.12.

\section{Introduction}

The dispersion dependence of the dielectric permittivity (DP) in the terahertz range in particular within the region of negative DP values today is a subject of the increased researchers' interest due to prospects to create materials with a negative refraction index (metamaterials) [1]. Negative refraction index media can be formed using the simultaneous availability of the negative dielectric permittivity $(\varepsilon)$ and magnetic permeability $(\mu)$. It is able to cause radical anomalies in propagation of electromagnetic waves, namely: plane-parallel plate shows focusing properties [2], light repulsion is replaced by light attraction [3]. However, after a long history of spectroscopic studies of the DP dispersion by using the infrared (IR) and Raman spectroscopy methods, it has not been given proper attention to the problem of the quantitative determination of a real band where DP has a negative value.

The region of negative DP values theoretically occurs always between the transverse $\omega_{T}$ and longitudinal $\omega_{L}$ phonons frequencies ( $\mathrm{T}-\mathrm{L}$ splitting). It is resulted using a simplified model without damping $(\Gamma=0)$ and based on the well-known Kurosawa ratio [4]. Indeed, within this approximation $\varepsilon(\omega) \rightarrow-\infty$ at $\omega \rightarrow \omega_{T}$, for any frequencies pair $\omega_{T}<\omega<\omega_{L}$. However, in the case when damping is present $(\Gamma>>0)$, the real part of DP cannot reach negative values. The effect of damping the polar vibrations on dispersive properties of the crystalline media has been studied in this work, and it has been shown that just the damping action plays a crucial role in shaping of the minimum achievable negative value of DP and width of the frequency area of its availability.

\section{Basic theory and problem statement}

The light interaction with polar vibrations of the crystal lattice is described in the semi-classical approach [5]. In this case, the dispersion dependence $\varepsilon(\omega)$ for crystals with $N_{k}$ vibration modes is determined by the general expression: 
$\varepsilon_{k}(\omega)=\varepsilon_{k}^{\infty}+\sum_{i=1}^{N_{k}} \frac{S_{i k}\left(\omega_{i k}^{T}\right)^{2}}{\left(\omega_{i k}^{T}\right)^{2}-\omega^{2}-i \omega \Gamma_{i}}$,

where $\omega_{i k}^{T}$ and $S_{i k}$ are, respectively, the transverse phonon frequencies and oscillator strength of the $i-$ th lattice vibrations with polarization along the $k$-th axis; $\Gamma_{i}$ is the damping constant; $\varepsilon_{k}^{\infty}$ is the main value of dielectric tensor at optical frequencies.

The components of the DP tensor (1) can be expressed in general by terms of the longitudinal $\omega_{i k}^{L}$ and the transverse $\omega_{i k}^{T}$ vibration frequencies as a Kurosawa ratio [4]:

$\varepsilon_{k}(\omega)=\varepsilon_{k}^{\infty} \prod_{i=1}^{N_{k}} \frac{\left(\omega_{i k}^{L}\right)^{2}-\omega^{2}}{\left(\omega_{i k}^{T}\right)^{2}-\omega^{2}}$,

which is actually a fair in the approximation of absence of phonon damping $\Gamma_{i}=0$. It is in this approximation:

$S_{i}=\varepsilon^{\infty}\left[\left(\frac{\omega_{i}^{L}}{\omega_{i}^{T}}\right)^{2}-1\right] \prod_{j \neq 1}^{N} \frac{\left(\omega_{j}^{L}\right)^{2}-\left(\omega_{i}^{T}\right)^{2}}{\left(\omega_{j}^{T}\right)^{2}-\left(\omega_{i}^{T}\right)^{2}}$,

where for simplicity it is omitted the index $k$ for polarization marking.

Within frameworks of the single-oscillator model, the dispersion dependence $\varepsilon(\omega)$ (1) and complex refraction index $\widetilde{n}=n-i \kappa$ can be written as:

$\widetilde{n}^{2}=\varepsilon(\omega)=\varepsilon^{\infty}+\frac{S \omega_{T}^{2}}{\omega_{T}^{2}-\omega^{2}-i \omega \Gamma}$.

Our analysis of the dispersion inherent to the dielectric permittivity in the case of non-zero damping is performed using the above Eq. (1) [6]. Exp. (4) describes the DP dispersion within the single-oscillator model, and it has been used as a base for our calculations. It allows to investigate the damping action on the negative DP region formation. The complex function $\varepsilon(\omega)(4)$ can be taken in the following form:

$\varepsilon(\omega)=\varepsilon^{\prime}(\omega)+i \varepsilon^{\prime \prime}(\omega)$.

The explicit form of $\varepsilon^{\prime}$ and $\varepsilon^{\prime \prime}$ can be found from the ratios (4) and (5):

$$
\left\{\begin{array}{l}
\varepsilon^{\prime}(\omega)=\varepsilon^{\infty}+\frac{S \omega_{T}^{2}\left(\omega_{T}^{2}-\omega^{2}\right)}{\left(\omega_{T}^{2}-\omega^{2}\right)^{2}+\omega^{2} \Gamma^{2}} \\
\varepsilon^{\prime \prime}(\omega)=\frac{S \omega_{T}^{2} \omega \Gamma}{\left(\omega_{T}^{2}-\omega^{2}\right)^{2}+\omega^{2} \Gamma^{2}}
\end{array}\right.
$$

The measured reflection coefficient $R$ at normal incidence is associated with the refraction index $n$ and extinction coefficient $\kappa$ as follows:
$R=\frac{(n-1)^{2}+\kappa^{2}}{(n+1)^{2}+\kappa^{2}}$.

Dispersion options $S, \omega_{T}$ and $\Gamma$ are measured directly from the Raman spectra or find by the numerical methods from the condition of the best approximation for the reflection spectrum curve. In the approximation when absorption is neglected, the frequency of longitudinal phonons $\omega_{L}$ can be found from the condition of the band zero contribution into the low frequency dielectric constant $\varepsilon_{0}$.

The relationship between material and spectroscopic parameters of the medium is described by the following ratio:

$\frac{\omega_{L}}{\omega_{T}}=\sqrt{\frac{\varepsilon_{0}}{\varepsilon^{\infty}}}$,

which is a known Lyddane-Sachs-Teller ratio [7].

Fig. 1 shows the reflection spectra $(a)$ and dispersion dependence of the DP real part $(b)$ for model crystalline medium. The dotted line describe the function (2) in the case when damping is absent $(\Gamma=0)$. It is damping the optical phonons that eliminates the gap in the curves for $\Gamma>0$, as shown in Fig. 1, and, in fact, it determines the anomalous dispersion of waves in the crystal and appearance of the region with the negative dielectric permittivity. The negative dielectric permittivity region, in accordance with the Kurosawa ratio (2), will be presented for any frequencies within the $\mathrm{T}-\mathrm{L}$ splitting region, and its boundaries will be matched to the frequencies of transverse $\omega_{T}$ and longitudinal $\omega_{L}$ oscillations. However, Fig. $1 \mathrm{~b}$ shows that the minimum value $\varepsilon_{\min }^{\prime}$ is increased with $\Gamma$, and the frequency band with negative DP is simultaneously narrowed. The state $\varepsilon_{\min }^{\prime}=0$ is achieved for certain "critical" damping $(\Gamma=0.085$ in Fig. $1 b)$, and the frequency band with negative DP completely disappears. It should be noted that the reflection spectra (Fig. 1a) does not contain any specific features for marking the presence or absence of negative DP area. Therefore, the quantitative analysis of the conditions for availability of the negative DP and the real band of its implementation are performed in this work using the physical and spectroscopic parameters of the crystal medium.

\section{Criterion for existence of frequency bands with a negative DP}

The dispersion dependence $\varepsilon(\omega)$ can be written as (4) in the simplest case for crystals with a single polar vibration. The oscillator strength is defined as $S=\varepsilon_{0}$ $-\varepsilon^{\infty}$ for this case.

It should satisfy the next condition at frequencies where the real part of DP (6) reaches its extreme value:

$\frac{\partial \varepsilon^{\prime}}{\partial \omega}=0$. 


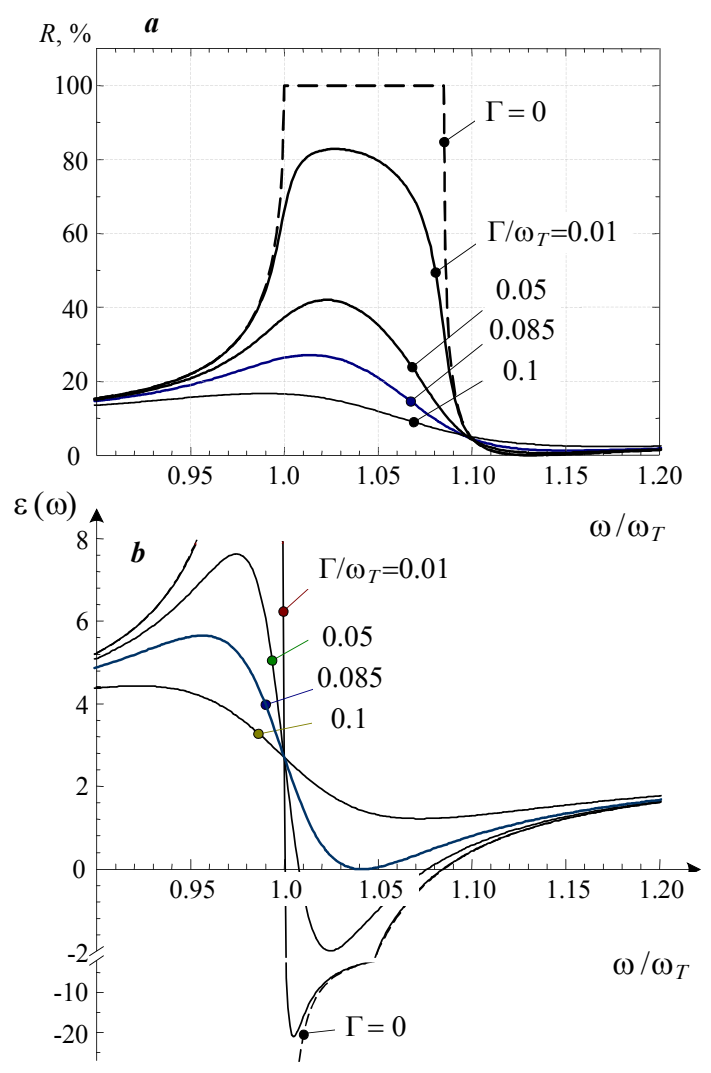

Fig. 1. Reflection spectra (a) and dispersion dependence of the real part of DP (b) for model crystalline medium. The dotted line illustrates an ideal case (Kurosawa ratio (2)), solid line - a real case of non-zero damping.

According to (9), after differentiating the first equation of system (6), and not rejecting the null common denominator, one can obtain an intermediate equation:

$$
\begin{aligned}
& -2 S \omega_{T}^{2} \omega\left(\omega_{T}^{2}-\omega^{2}\right)^{2}-2 S \omega_{T}^{2} \omega^{3} \Gamma^{2}+ \\
& +4 S \omega_{T}^{2} \omega\left(\omega_{T}^{2}-\omega^{2}\right)^{2}-2 S \omega_{T}^{4} \omega \Gamma^{2}+2 S \omega_{T}^{2} \omega^{3} \Gamma^{2}=0 .
\end{aligned}
$$

After simplification, the expression (10) will look as:

$$
\left(\omega_{T}^{2}-\omega^{2}\right)^{2}=\omega_{T}^{2} \Gamma^{2} .
$$

The frequency position of the $\varepsilon^{\prime}(\omega)$ minimum is determined by one of the equation (11) roots, namely it is belonged to the interval of frequencies $\omega>\omega_{T}$. Moreover, the point $\omega$ will be the minimum point $\left(\omega_{\min }\right)$, when it is equal:

$\omega_{\min }=\sqrt{\omega_{T}^{2}+\omega_{T} \Gamma}$.

After substitution $\omega_{\min }$ to the first equation of the system (6), we obtain the minimum value of the dielectric function $\varepsilon^{\prime}(\omega)$. Note that by (12) the frequency position of the minimum and the minimum value of $\varepsilon^{\prime}(\omega)(6)$ depend on the damping parameter $\Gamma$ (Fig. 1b). So, for availability of the negative permittivity region, where $\varepsilon^{\prime}(\omega)<0$, it is necessary that:

$\varepsilon^{\prime}\left(\omega_{\min }\right)<0$, where

$$
\varepsilon^{\prime}\left(\omega_{\min }\right)=\varepsilon_{\min }^{\prime}=\varepsilon^{\infty}-\frac{S \omega_{T}^{2}}{2 \omega_{T} \Gamma+\Gamma^{2}},
$$

whence the inequality directly follows:

$$
\Gamma^{2}+2 \omega_{T} \Gamma-\frac{S}{\varepsilon^{\infty}} \omega_{T}^{2}<0 .
$$

Changes of the minimum value of DP $\left(\varepsilon_{\min }^{\prime}\right)$ as a function of the damping constant $\Gamma$ observed in Fig. 1 are fully described by the analytical dependence (13). In particular, if $\Gamma \rightarrow 0$ in Eq. (13), then $\varepsilon_{\min }^{\prime} \rightarrow-\infty$ like to that in the Kurosawa ratio (2).

The inequality (14) for values of $\Gamma>0$ leads to the following conditions:

$$
\Gamma<\omega_{T}\left(\sqrt{\frac{\varepsilon_{0}}{\varepsilon^{\infty}}}-1\right),
$$

and it gives a simple quantitative criterion for existence of frequency bands with negative DP within the singleoscillator crystal model. It is based on the ratio of damping constant phonon with the frequency $\omega_{T}$ to such material parameters of crystal as $\varepsilon_{0}$ and $\varepsilon^{\infty}$.

The criterion (15) can be represented through spectroscopic parameters of the crystal medium, if we apply to it the ratio of Lyddane-Sachs-Teller (8). Then, the condition of availability of the negative DP region takes the following form:

$$
\Gamma<\left(\omega_{L}-\omega_{T}\right) \text { or } \frac{\Gamma}{\omega_{L}-\omega_{T}}<1 .
$$

In fact, existence of the negative dielectric permittivity region until the phonon damping constant does not exceed $\mathrm{T}-\mathrm{L}$ splitting for this phonon is the immediate consequence of (16).

Thus, the critical damping $\Gamma_{c r}$ should be determined using (15) and (16). It corresponds to the upper boundary phonon damping and restricts the existence of negative DP in the crystal:

$\frac{\Gamma_{c r}}{\omega_{T}}=\sqrt{\frac{\varepsilon_{0}}{\varepsilon^{\infty}}}-1=\frac{\omega_{L}}{\omega_{T}}-1$ or $\Gamma_{c r}=\omega_{L}-\omega_{T}$.

The dispersion dependence $\varepsilon^{\prime}(\omega)$ for critical damping at $\Gamma / \omega_{T}=0.085$ is illustrated by the curve in Fig. 1b, if the minimum of real part for DP is equal to zero.

If $\Gamma<\Gamma_{c r}$, then the frequency band with negative DP values is formed around the minimum of DP within $\mathrm{T}-\mathrm{L}$ splitting frequencies of these oscillations. And 
vice versa, when $\Gamma>\Gamma_{c r}\left(\Gamma / \omega_{T}=0.1\right.$ in Fig. 1b) the function $\varepsilon^{\prime}(\omega)$ is positive, including the band of residual rays $(\mathrm{T}-\mathrm{L})$.

\section{Real frequency band with negative DP}

The frequency range of negative DP area really does not match to the $\mathrm{T}-\mathrm{L}$ splitting, unlike the idealized case of no damping, which is described by the Kurosawa ratio (2). The negative DP area is shrunk relative to $\mathrm{T}-\mathrm{L}$ splitting up to its complete disappearance due to increase of the damping constant as shown in Fig. $1 \mathrm{~b}$. Therefore, we have studied behavior of the frequency band with negative DP as a function of the damping constant.

Let us introduce the notation of the frequencies: $\omega_{-}$is the frequency when DP begins to take negative values, and $\omega_{+}$is the frequency when DP goes out from negative region. If for expression (3) consider the case of single-oscillator model $(N=1)$ and substitute the resulting value for the oscillator strength in the first equation of the system (6), then:

$$
\varepsilon^{\prime}(\omega)=\varepsilon^{\infty}\left[1+\frac{\left(\omega^{2}-\omega_{T}^{2}\right)\left(\omega_{T}^{2}-\omega_{L}^{2}\right)}{\Gamma^{2} \omega^{2}+\left(\omega^{2}-\omega_{T}^{2}\right)^{2}}\right] .
$$

The frequencies $\omega_{-}$and $\omega_{+}$are found from terms of equality of the real part of DP, described with the expression (18), to zero $\varepsilon^{\prime}(\omega)=0$. After simple mathematical transformations, rejecting no physical variations, we obtain:

$\omega_{ \pm}=\frac{1}{\sqrt{2}}\left(\omega_{L}^{2}+\omega_{T}^{2}-\Gamma^{2} \pm \sqrt{\left(\omega_{L}^{2}+\omega_{T}^{2}-\Gamma^{2}\right)^{2}-4 \omega_{L}^{2} \omega_{T}^{2}}\right)^{1 / 2}$

Let $\Delta \omega=\omega_{+}-\omega_{-}$is the real frequency band of negative DP. An explicit expression for $\Delta \omega$ may be easily found using the relations (19), so we have:

$\Delta \omega=\left(\omega_{L}-\omega_{T}\right) \sqrt{1-\left(\frac{\Gamma}{\omega_{L}-\omega_{T}}\right)^{2}}$.

The expression (20) is the generalization of the no damping idealized case as if $\Gamma=0$, then $\Delta \omega=\omega_{L}-\omega_{T}$. Similarly, if in expressions (19) to direct the damping constant to zero, we get: $\omega_{-}=\omega_{T}$, and $\omega_{+}=\omega_{L}$.

Our analysis shows that a large number of crystals with one pronounced vibration in the infrared region, such as classic items of alkali-halide crystals $(\mathrm{NaCl}$, $\mathrm{KBr}, \mathrm{NaF}, \mathrm{LiF}$, and many others) in the anomalous dispersion region reaches sufficiently high in modulus negative values of DP. For these crystals, the difference $\omega_{L}-\omega_{T}$ reached tens of $\mathrm{THz}$, whereas the damping constant $\Gamma$ was typically less then one $\mathrm{THz}$ at room and low temperatures. Therefore, the ratio $\Gamma /\left(\omega_{L}-\omega_{T}\right)<0.1$ and according to (16) ensures the existence of high in modulus negative values of DP observed in practice, moreover the frequency range with $\varepsilon^{\prime}(\omega)<0$ almost coincides with the interval of $\mathrm{T}-\mathrm{L}$ splitting, according to (20). However, the absence of negative DP with relative damping $\Gamma /\left(\omega_{L}-\omega_{T}\right)>1$ has been recently found by us in some crystalline modifications of boron nitride $[8,9]$.

In addition, there are a class of non-magnetic media, in particular nonlinear crystals, where the parameters $\Gamma, \omega_{T}, \omega_{L}$ may be directly measured from the Raman spectra. The existence and width of range of negative DP in $\mathrm{LiTaO}_{3}$ were quantitatively investigated in this work.

\section{Negative dielectric permittivity in $\mathrm{LiTaO}_{3}$ crystal}

$\mathrm{LiTaO}_{3}$ has 30 vibrational degrees of freedom in the ferroelectric phase, which are distributed by the types of symmetry: $5 A_{1}+5 A_{2}+10 E$, moreover $E$ variations are double degenerate. Acoustic phonons belonging to the representations $A_{1}$ - and $E$-type, and vibrations from $A_{2}$ - type do not appear both in IR and Raman spectra.

The feature of the $A_{1}$-type and $E$-type vibrations are as follows: they are active both in Raman spectra and IR reflection spectra, and this crystal was chosen for our research.

The polar vibration parameters of crystal lithium tantalate for parallel ( $A_{1}-$ type) and perpendicular ( $E$-type) polarizations are shown in Table, and they are measured from the Raman spectra. Our calculated parameters are presented in columns 5-9 for each oscillation, namely: $\mathrm{T}-\mathrm{L}$ splitting, normalized to $\mathrm{T}-\mathrm{L}$ splitting damping (16), frequencies $\omega_{+}$and $\omega_{-}$ (19), the frequency width of the negative DP area (20). The minimum negative DP value ( $\varepsilon_{\min }^{\prime}$, column 10) is calculated directly from (6), as in (13) parameter $\varepsilon^{\infty}$ is not monosemantic in the case of complex phonon spectrum.

The frequency dispersion of DP for $\mathrm{LiTaO}_{3}$ crystal is shown in Fig. 2 for both polarizations. The oscillation number in Fig. 2 corresponds to the number in Table. All vibrations of the parallel polarization show negative DP areas, in accordance to quantitative data calculated using our criteria (16), and they can be seen in Fig. 2. The vibrations $A_{1}$ and $A_{2}$ have very "deep" negative DP areas: $\varepsilon_{\text {min }}^{\prime}=-119$ and $\varepsilon_{\text {min }}^{\prime}=-40$, respectively, and in their absolute values are higher than the DP values at low $\left(\varepsilon_{0}=30.24\right)$ and high $\left(\varepsilon^{\infty}=4.15\right)$ frequencies. The vibrations $A_{1}$ and $A_{2}$ create the common region of negative DP from 6.06 to $10.39 \mathrm{THz}$ as it is also shown in Fig. 2. The real frequency bands of negative DP values $\Delta \omega$ (see Table) are calculated using Eq. (20) for all the vibrations in $\mathrm{LiTaO}_{3}$ crystal ( $A_{1}$-type), and they 
Table. Polar vibration parameters for crystal $\mathrm{LiTaO}_{3}$.

\begin{tabular}{|c|c|c|c|c|c|c|c|c|c|}
\hline № & $\begin{array}{c}\omega_{L} \\
(\mathrm{THz})\end{array}$ & $\begin{array}{c}\omega_{T} \\
(\mathrm{THz})\end{array}$ & $\begin{array}{c}\Gamma \\
(\mathrm{THz})\end{array}$ & $\begin{array}{c}\omega_{L}-\omega_{T} \\
(\mathrm{THz})\end{array}$ & $\frac{\Gamma}{\omega_{L}-\omega_{T}}$ & $\begin{array}{c}\omega_{+} \\
(\mathrm{THz})\end{array}$ & $\begin{array}{c}\omega_{-} \\
(\mathrm{THz})\end{array}$ & $\begin{array}{c}\Delta \omega \\
(\mathrm{THz})\end{array}$ & $\varepsilon_{\min }^{\prime}$ \\
\hline 1 & 2 & 3 & 4 & 5 & 6 & 7 & 8 & 9 & 10 \\
\hline \multicolumn{10}{|c|}{$A_{1}$-type $\varepsilon^{\infty}=4.15, \varepsilon_{0}=30.24$} \\
\hline$A_{1}$ & 7.35 & 6.03 & 0.45 & 1.32 & 0.34 & 7.31 & 6.06 & 1.25 & -119 \\
\hline$A_{2}$ & 10.41 & 7.59 & 0.45 & 2.82 & 0.16 & 10.39 & 7.61 & 2.78 & -40 \\
\hline$A_{3}$ & 11.97 & 10.68 & 0.24 & 1.29 & 0.19 & 11.96 & 10.69 & 1.27 & -7.6 \\
\hline$A_{4}$ & 25.92 & 18 & 0.24 & 7.92 & 0.03 & 25.92 & 18.00 & 7.92 & -107 \\
\hline \multicolumn{10}{|c|}{$E$-type $\varepsilon^{\infty}=4.13, \varepsilon_{0}=35.78$} \\
\hline$E_{1}$ & 2.4 & 2.22 & 0.57 & 0.18 & 3.17 & \multicolumn{4}{|c|}{ No negative DP area } \\
\hline$E_{2}$ & 4.89 & 4.2 & 0.18 & 0.69 & 0.26 & 4.87 & 4.21 & 0.66 & -101 \\
\hline$E_{3}$ & 7.44 & 6.18 & 0.18 & 1.26 & 0.14 & 7.43 & 6.19 & 1.24 & -130 \\
\hline$E_{4}$ & 8.34 & 7.53 & 0.3 & 0.81 & 0.37 & 8.31 & 7.56 & 0.75 & -9.5 \\
\hline$E_{5}$ & 9.54 & 9.48 & 0.18 & 0.06 & 3.00 & \multicolumn{4}{|c|}{ No negative DP area } \\
\hline$E_{6}$ & 13.56 & 11.49 & 0.36 & 2.07 & 0.17 & 13.54 & 11.51 & 2.03 & -47 \\
\hline$E_{7}$ & 14.22 & 13.86 & 0.18 & 0.36 & 0.50 & 14.20 & 13.88 & 0.32 & -2.7 \\
\hline$E_{8}$ & 19.44 & 17.88 & 0.33 & 1.56 & 0.21 & 19.42 & 17.90 & 1.52 & -53 \\
\hline$E_{9}$ & 26.1 & 19.86 & 0.45 & 6.24 & 0.07 & 26.09 & 19.87 & 6.22 & -17 \\
\hline
\end{tabular}

Note. Column \#1 is the vibration number; \#2 and \#3 are frequencies of longitudinal and transverse vibrations, respectively; \#4 and \#5 are the damping constant $(\Gamma)$ and $\mathrm{T}-\mathrm{L}$ splitting; \#6 is damping normalized to $\mathrm{T}-\mathrm{L}$ splitting; \#7 and \#8 are the frequencies at which the DP comes from the negative region $\left(\omega_{+}\right)$and begins to take negative values $\left(\omega_{-}\right)$; $\# 9$ is the range width for negative DP; \#10 is the minimum negative DP value.

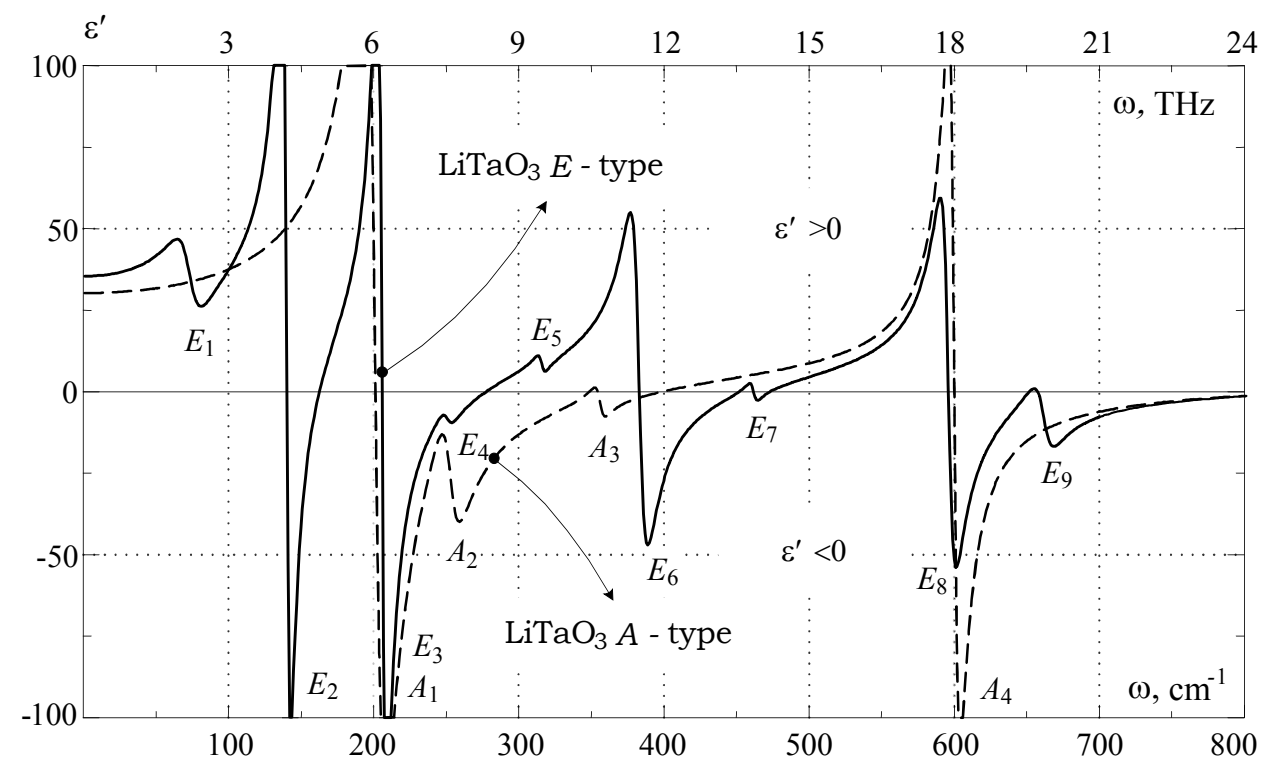

Fig. 2. Frequency dispersion of the real part of $\mathrm{DP}$ for $\mathrm{LiTaO}_{3}$ crystal in the cases of parallel $\left(A_{1}-\right.$ type, dotted line $)$ and perpendicular ( $\mathrm{E}$ - type, solid line) polarizations.

are very close to the $\mathrm{T}-\mathrm{L}$ splitting due to the fact that relative damping is much smaller than unity. So, as Eq. (20) was derived for the single-oscillator crystal model, the vibrations with common negative DP areas can cause significant uncertainties. Let us consider, for example, vibrations $E_{3}$ and $E_{4}$ in $\mathrm{LiTaO}_{3}$ crystal for perpendicular polarization. These vibrations have a common negative DP area in accord with Fig. 2, however, quantitative data (Table, columns 7-9) are indicative of the splitting of negative DP for $E_{3}$ and $E_{4}$ vibrations by the positive DP band with the $0.12-\mathrm{THz}$ width. Exact determination of the frequency band where DP gets negative values for the merge areas case can be calculated using Eq. (19) considering $\omega_{-}$for the former oscillation and $\omega_{+}$for the latter oscillation, respectively.

Absence of the negative DP regions for $\omega_{T}=2.22 \mathrm{THz}$ and $\omega_{T}=9.48 \mathrm{THz}$ of perpendicular polarization (see Fig. 2, vibrations $E_{1}$ and $E_{5}$ ) also corresponds to the table data and criterion (16), because 
the relative damping value in the order of 3. In this case, the ratio (20) returns the complex roots, which is logical because the frequency dispersion of the real part of DP does not cross zero. According to (16) and quantitative data in Table, all other phonon oscillations have negative DP area, as we can see in Fig. 2.

The $A_{1}$-type has no phonon vibrations in the lowfrequency region $2.1-5.1 \mathrm{THz}$, unlike $E$-type that has two vibrations, one of them $\left(E_{2}\right)$ reaches large negative values $\left(\varepsilon_{\min }^{\prime}=-101\right)$. It should be noted that both polarizations have two common frequency regions with negative DP. There are two characteristic vibrations for $A_{1}$-type $\left(A_{1}, A_{2}\right)$ and two vibrations for $E$-type $\left(E_{3}, E_{4}\right)$ in the range from 6.06 to $8.28 \mathrm{THz}$. One oscillation of $A_{1}$-type has a pronounced negative DP area $\left(\varepsilon_{\min }^{\prime}=-107\right), E$-type is separated by two close vibrations with two negative bands with minimum values -53 and -17 in the high-frequency region 17.88 up to $26.07 \mathrm{THz}$, respectively.

\section{Conclusions}

The research results of physical conditions providing existence of the region with negative DP values in real crystalline medium have been presented in this work. It has been shown that the existence of negative DP in crystals essentially depends on damping the polar phonons. The quantitative criterion (16) for the existence of frequency bands with negative DP has been obtained on the basis of the single-oscillator crystal model. The analytical expressions (19), (20) for calculation of the frequency bands with negative DP have been obtained. The estimation of narrowing the negative DP area relatively to the $\mathrm{T}-\mathrm{L}$ frequency splitting as a function of damping has been presented.

There are two areas (from 6.06 to $8.28 \mathrm{THz}$ and from 17.88 to $26.07 \mathrm{THz}$ ) in $\mathrm{LiTaO}_{3}$ crystal where both components of DP tensor acquire negative values. The analysis confirms the applicability of the criterion (16) for crystals with a relatively complex spectrum of polar phonons. It has been shown that the negative DP band can be calculated using (20) with a high accuracy in the case of separate areas and allows in the first approximation to estimate the frequency band for the common areas of negative DP.
The analytical dependences between the basic parameters of crystals expressed by Eqs (16), (19), and (20) may be useful when analyzing the negative regions of DP in crystalline media with rather complicated phonon spectrum.

\section{References}

1. V.G. Veselago, Electrodynamics of materials with negative refractive index // Physics - Uspekhi, 173 (7), p. 790-794 (2003).

2. V.G. Veselago, Formulating Fermat's principle for light traveling in materials with negative refraction // Physics - Uspekhi, 172 (10), p. 1215-1218 (2002).

3. V.G. Veselago, Energy, momentum and mass transfer by an electromagnetic wave in a negative refraction medium // Physics - Uspekhi, 179 (6), p. 689-694 (2009).

4. T. Kurosawa, Polarization waves in solids // $J$. Phys. Soc. Jap. 16 (9), p. 1288-1308 (1961).

5. P.A. Korotkov, and G.S. Felinskyi, Research of negative dielectric permeability area in the media without inversion center // Bull. Kyiv Univ.: ser. Phys. \& Math. No.2, p. 162-171 (2008), in Ukrainian.

6. S.G. Felinskyi, P.A. Korotkov, and G.S. Felinskyi, Criterion of the existence region of negative dielectric permittivity at the polar oscillations frequencies in crystals // Bull. Kyiv Univ.: ser. Phys. \& Math. No.1, p. 191-196 (2010), in Ukrainian.

7. R. Lyddane, R.G. Sachs, E. Teller, On the polar vibrations of alkali halides // Phys. Rev. 59, p. 673676 (1941).

8. S.G. Felinskyi, P.A. Korotkov, and G.S. Felinskyi, Negative dielectric function in anisotropic modifications of boron nitride // New technologies, No.2, p. 51-57 (2010), in Ukrainian.

9. S.G. Felinskyi, P.A. Korotkov, G.S. Felinskyi, Terahertz properties and the negative dielectric regions in boron nitride // IEEE Intern. Workshop on THz Radiation: Basic Research \& Applications (TERA 2010), Sevastopol, 2010, September 12-14, p. 265-266. 